\title{
Integrated design for integrated photonics: from the physical to the circuit level and back
}

\author{
Wim Bogaerts, Yanlu Li, Shibnath Pathak, Alfonso Ruocco, Martin Fiers, \\ Antonio Ribeiro, Emmanuel Lambert, Pieter Dumon \\ Ghent University - imec, Photonics Research Group, Department of Information Technology, \\ Center for Nano and Biophotonics, Sint-Pietersnieuwstraat 41, 9000 Gent, Belgium
}

\begin{abstract}
Silicon photonics is maturing rapidly on a technology basis, but design challenges are still prevalent. We discuss these challenges and explain how design of photonic integrated circuits needs to be handled on both the circuit as on the physical level. We also present a number of tools based on the IPKISS design framework.
\end{abstract}

Keywords: Photonic Integrated Circuits, Design, Simulation

\section{INTRODUCTION}

Silicon Photonics is rapidly gaining industrial traction, the fabrication technology becomes more mature and device demonstrations start to meet functional specs for real-life applications. The scaling of silicon optical waveguides to submicron dimensions also opens the opportunity for very compact chips that can accommodate unprecedented levels of complexity in a photonic circuit. But at the same time, the submicron dimensions and high index contrast introduce low tolerances to fabrication variations. This combination of high complexity with low tolerances sounds like a recipe for a low yield. And this is difficult to counter on the design side, because the design process for integrated photonics has not evolved as rapidly as the technology base for silicon photonics.

Photonic design is still very much a physical design activity, especially in silicon photonics, where the high refractive index contrast makes most common approximations for optical simulations useless. Full-vectorial brute-force electromagnetic number crunching of physical geometries is still the most applied design method for individual building blocks, ${ }^{1-5}$ or alternatives based on mode expansion ${ }^{6,7}$ and propagation. ${ }^{8}$ These methods cannot scale up to large, complex circuits, which need to be designed at circuit level. But at this level, suitable simulation tools are only just emerging. ${ }^{9-12}$ Such circuit simulators are based on behavioral models to efficiently simulate many circuit elements.

As circuit simulators and physical simulators are generally based on very different principles, they are often used separately. However, for silicon photonics design, where there is still a very strong need for physical simulation while at the same time larger circuits need to be simulated, these two levels of simulation should be more tightly integrated. For example, a circuit simulation might require a detailed physical simulation of a certain building block to establish the validity of a model or calculate the transmission. In an integrated design and simulation flow, such a simulation could be triggered automatically. At this moment, only a few tools offer this capability in a limited way. ${ }^{11,13,14}$

Photonic design tools have historically evolved apart from the electronic design automation (EDA) ecosystem, but with the advent of silicon photonics this is also about to change. Silicon photonic circuits will, for most applications, require complex control circuitry, and at that point electronic-photonic codesign becomes a necessity, whether the photonic and electronic circuits are collocated on the same die or on separate chips. Electronic design frameworks rely very strongly on parametric building blocks (PCells), a concept which has also been introduced in photonic design environments ${ }^{13,15}$

We present IPKISS, ${ }^{16,17}$ a parametric design framework for photonic integrated circuits. ${ }^{16}$ It is a componentcentric framework which allows the designer to perform several design and simulation activities from within the

Further author information: (Send correspondence to W.B.)

W.B.: E-mail: wim.bogaerts@intec.ugent.be, Telephone: +32 92643324 


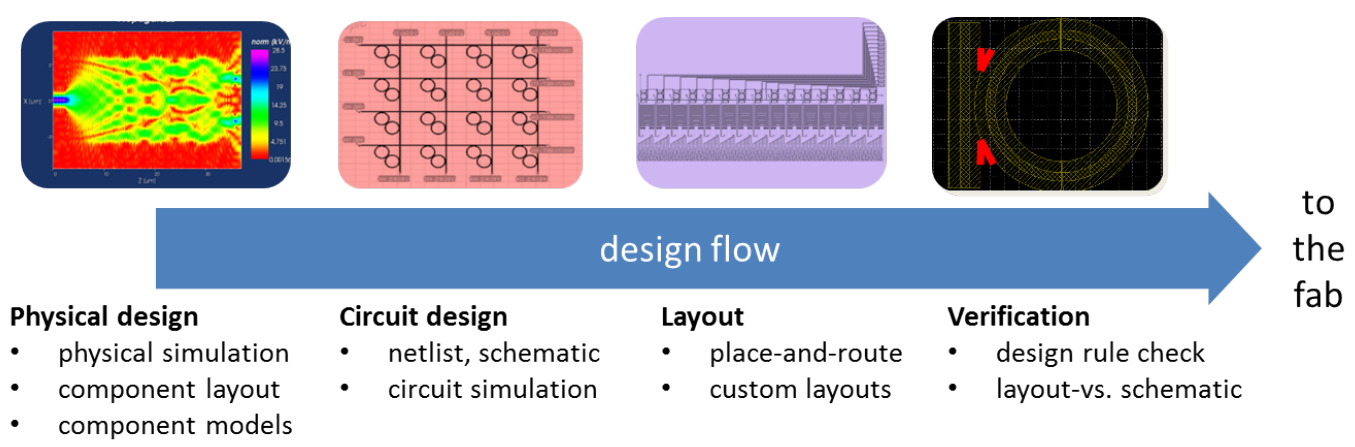

Figure 1. Design flow for photonic integrated circuits.

same component library, and generate different output based on the same component definition. As we will discuss further, we can define a component once in IPKISS, and from that definition launch an 2D or 3D FDTD simulation, an eigenmode expansion simulation, a circuit simulation, but also generate a mask layout for the component and the circuit. Also, integration with custom simulation tools and models is relatively easy, as the framework interface is open. This way, a bridge is laid between the physical design and the circuit design, and the designer can combine the strengths of different methods.

In this paper, we will discuss how IPKISS can facilitate the design flow of silicon photonics. First, we will take a closer look at the different steps taken in this design flow, from the physical design to the circuit level and finally back to the physical layout and its verification. Then, we will discuss the IPKISS framework in more detail and how it is used in each step of this process. Finally, we will illustrate the strength of this method with a number of challenging design examples.

\section{SILICON PHOTONIC DESIGN FLOW}

To design a photonic integrated circuit, most designers will have to go through at least 4 phases before the design can be sent out for fabrication. This is illustrated in Fig. 1.

Component design First of all, individual building blocks need to be designed according to initial specifications. This is typically a physical design step involving mask layout and electromagnetic modeling. Today, component design is still very much a part of actual photonic circuit design. It is expected however, that in the future this part of the design flow will be largely done by the fab, which will use it to define standard component for its design kit. Still, full-custom component design for circuits will still be an important activity in the foreseeable future.

Circuit design The designed components are linked together in a schematic, and the response of the resulting circuit is simulated. Depending on the type of photonic components in the circuit and the targeted application, this simulation can become very complex. This is because photonic behavior has a richness that surpasses that of electronics, requiring the modeling of phase-coherent signals over a wide wavelength range.

Circuit layout The high-level description of the circuit is translated into a mask layout for fabrication. Photonic components have a more complex layout that electronic building blocks. Photonic waveguides follow curvilinear shapes with smooth bends, and impose different requirements on alignment and pattern fidelity than electronic designs.

Verification The layout is checked for violations of design rules, and whether it matches the functional description of the circuit. For this design rule checking (DRC), verification tools from the electronics design world are used, ${ }^{18}$ although some tools support DRC specifically for photonics. ${ }^{19}$ But these are not (yet) well equipped to handle the smooth curves of photonics. Also, extracting functional information and interconnectivity from a photonic layout (layout-vs-schematic or LVS) is very different than for an electronic layout. 
As already explained in the introduction, these steps are usually not very well integrated for a photonic design flow, and often the designer will need to redo a significant part of the work to redefine components in different tools. This makes such a design flow prone to casual errors due to wrongly copying design parameters between different steps in the flow. Still, some design tools do integrate some steps in the flow, such as a link between physical and circuit simulation ${ }^{11,14}$ or between circuit simulation and layout. ${ }^{3,20}$

\section{IPKISS: A PARAMETRIC DESIGN FRAMEWORK}

IPKISS is a photonic design framework developed at Ghent University and imec. Its origins date back to 2001 when research in silicon photonics exposed the needs for tools to generate complex waveguide circuit layouts in a parametric way (e.g. to generate designs with parameter sweeps on some circuit elements). At that point, the decision was made to implement this tool as a scripting framework in Python, because of the flexibility and readability of the language and the availability of many high-performance scientific libraries. ${ }^{21}$ With IPKISS, the photonics research group has generated hundreds of designs of silicon photonic integrated circuits, and a large library of parametric building blocks was constructed, together with the layer settings to generate complex GDII files. Since then, IPKISS has evolved from a flexible GDSII generator to a broader component-oriented design framework. At the core is a library manager that allows users to define parametric building blocks. Building blocks can be hierarchical, which means they can contain other 'child' building blocks and connect them together into a (sub)circuit. In fact, complete circuits are treated in much the same way as atomic building blocks which do not have children. To these building blocks, different representations (views) can be added. Mask layout is only one of those. Other such views include an input-output ports, interconnectivity matrix (or netlist), 3D geometry, and interfaces to simulation tools for physical and circuit simulation.

IPKISS itself does not perform simulations, but the python scripting framework makes it easy to interface IPKISS to other simulators. The framework contains a set of generic interface layers to simulators, that can be extended to match a specific tool. For example, there is a generic FDTD layer, which is then implemented for MIT's open-source MEEP simulator, ${ }^{1}$ but it could just as well be used for another FDTD simulator, either commercial or open source. Similarly, IPKISS interfaces with an in-house developed mode solver, but also with CAMFR, an eigenmode expansion tool. ${ }^{7}$ For circuit simulators IPKISS has a link to Caphe, ${ }^{9,22}$ a powerful time and frequency domain optical circuit simulator, but the openness of the framework allows the use of other simulators as well.

IPKISS itself manages the interaction between the views and the associated tools. This, way, views can be made to depend on one another. One workflow which is predefined in IPKISS is illustrated in Fig. 2. A component (here, a $3 \mathrm{~dB}$ splitter based on a multi-mode interferometer) is defined with a number of parameters. This can be a predefined component, or a user-defined component. In this example, we need to define a mask layout for this component, consisting of a set of polygons on the correct layers, such that the component can be fabricated with lithography. The definition of these layers is stored in a Technology File, which contains the specifics of the fabrication technology. Base on this mask layout, IPKISS can now generate other views. If the Technology file specifies a fabrication process, IPKISS can apply the fabrication flow to the mask layout, which results in a 3D distribution of materials. This built-in virtual fabrication engine generates an approximate geometry of material stacks, which cannot capture the full physics of the actual fabrication, but interfacing IPKISS to alternative (commercial) TCAD simulators is definitely possible (but not implemented at this time). The 3-D geometry can be used directly to define the simulation volume for a physical simulation. If the user has also specified input and output ports, the simulation volume could be supplied with the necessary excitation sources and monitors at the inputs and outputs.

Based on this information, IPKISS can launch a physical simulation to extract the transmission from one input port to all outputs, and this simulation can be repeated (if needed) to extract a complete scatter matrix (S-matrix) of the component. the choice of physical simulation engine can depend on the component and could involve mode-expansion based techniques or FDTD. Such simulations could be controlled by an optimization algorithm to tailor the response of a component. ${ }^{23}$ This is illustrated further in one of the examples. The resulting S-matrix can also be used in a circuit model of the component and invoked when the component is used in a circuit simulator such as Caphe. These heavy physical simulations can be done in advance to extract and store such a circuit model, or they can be executed on demand when they are needed. The former can be 


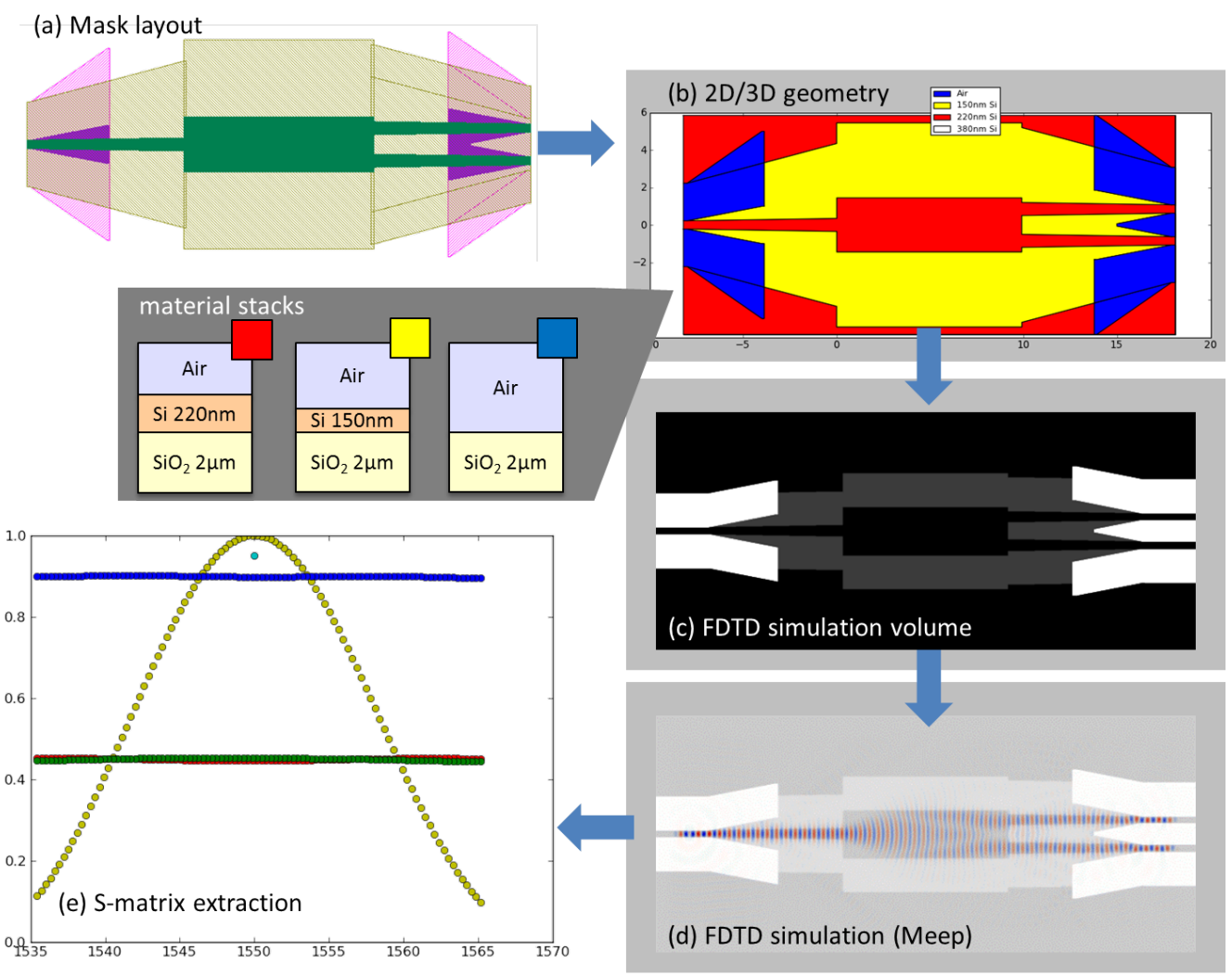

Figure 2. Views in IPKISS can be derived from one another. Example based on an multi-mode interferometer (MMI) for 3dB-splitting. (a) We can start from a mask layout (a) to generate a 2D of 3D material distribution (b) based on fabrication information. This geometry can be exported to a refractive index distribution (c) for simulation in an electromagnetic solver such as MEEP (d). From the output (e) a scatter matrix can be extracted.

desirable when many heavy simulations are needed, while the latter method is fine for occasional simulations or when the component parameters exceed the limits of the available circuit model. In general, the physical simulation will significantly slow down the circuit simulation, especially when many components need to be simulated.

IPKISS does not provide a solution for verification of design rules. Technology settings allow the user to specify design rules and use the preset values in the design to preempt design rule violations, but no hard checking is performed by IPKISS. However, IPKISS supplies export routines to generate design rule check decks for Calibre, the most widely used DRC engine for the verification of electronic IC designs. ${ }^{18}$

Fig. 3 shows a code example of how a designer can define custom components in Python. In this example, a directional coupler is defined. Every component in an IPKISS library is defined as a python class, and all programming functionality that works for Python classes can be used. These are inherently parametric objects, and we supply a shorthand syntax to describe all the parameters (in this example 5). From that point on, we can add views to the component by implementing methods. For example, the define_elements method will generate a mask layout.

IPKISS is not a design and simulation tool like many commercial solutions that are available. It is conceived as a scripting framework: its interface with the designer is through code written in Python, and as such there is not point-and-click graphical user interface (GUI) to guide the user through the design process. While this significantly raises the entry barrier to start using the framework, it is our opinion that it increases productivity in the long run. The scripting interface makes ad-hoc design somewhat more difficult, but facilitates the reuse and improvement of existing designs, using standard programming techniques such as class inheritance: complex 


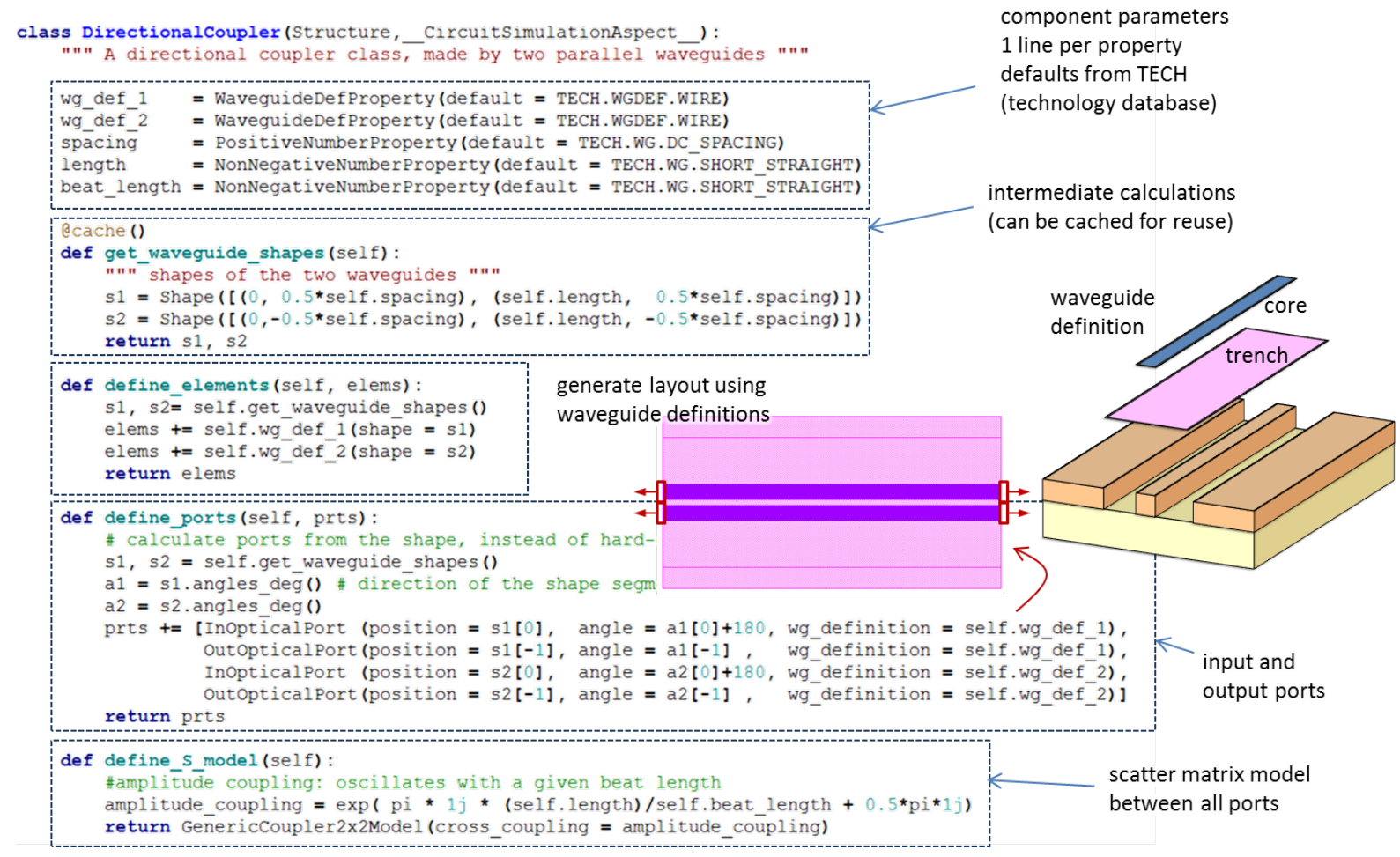

Figure 3. An example of a component definition in IPKISS, with parameters, layout generation, input/output ports and scatter matrix model. Additional aspects are added by defining additional functions.

components can inherit functionality from simpler, previously defined components. Also, automation of certain processes, such as parameter sweeps or optimization routines, comes naturally in a pure scripting environment. If a comparison is made electronic design automation, we see that graphical user interfaces play an important role, but a large fraction of electronic IC design is powered by a tremendous amount of scripting 'glue' in SKILL or Tcl. As such, scripting is a widely accepted method for complex circuit design.

As IPKISS has been in development and use for over 10 years, a sizable library of building blocks for silicon photonic circuits has been constructed. This includes waveguides ${ }^{24}$ spectral filters ${ }^{25}$ resonators ${ }^{26}$ photonic crystal devices, fiber coupler interfaces, ${ }^{27}$ but also active devices such as silicon modulators. ${ }^{28}$ The tool also supports design for the multi-project-wafer service ePIXfab. ${ }^{29}$ IPKISS can be accessed as open source under GPL-v2 license. ${ }^{16}$

\section{EXAMPLES}

Below we discuss a few different examples to illustrate the benefits of a flow where the different levels of design and abstraction can interact.

\subsection{Optimizing a 3dB combiner for reduced back-reflection}

A first example is a simple $3 \mathrm{~dB}$ combiner, consisting of a the multi-mode interferometer from Fig. 2, which is defined by a shallow etch in the silicon. ${ }^{25}$ While this device has a quite good efficiency in transmission (about $0.3 \mathrm{~dB}$ insertion loss), it does have a non-negligible reflection. When used in a circuit which requires bidirectional propagation, such as a laser-doppler vibrometer, ${ }^{30}$ these reflections cause spurious interferences in the return signal. We investigated the source of the backreflection by running an FDTD simulation on the standard MMI component. This is a straightforward operation in IPKISS which requires a few lines of script code. From that, we saw that the corners at the beginning of the exit waveguide contribute the most to the scattering and also the backreflection (4). 
(a)

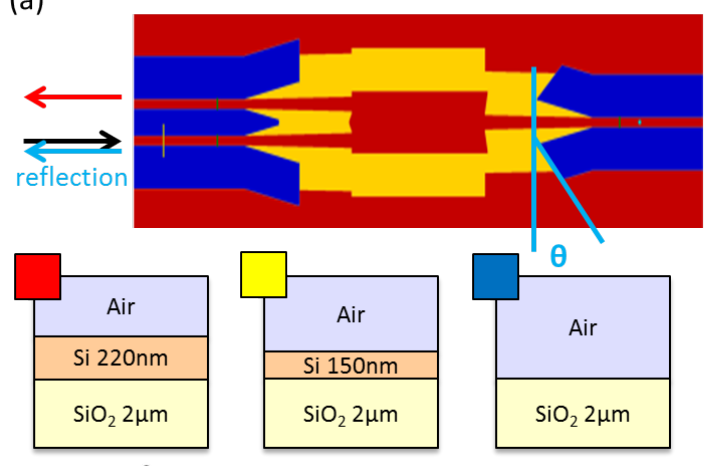

(b)
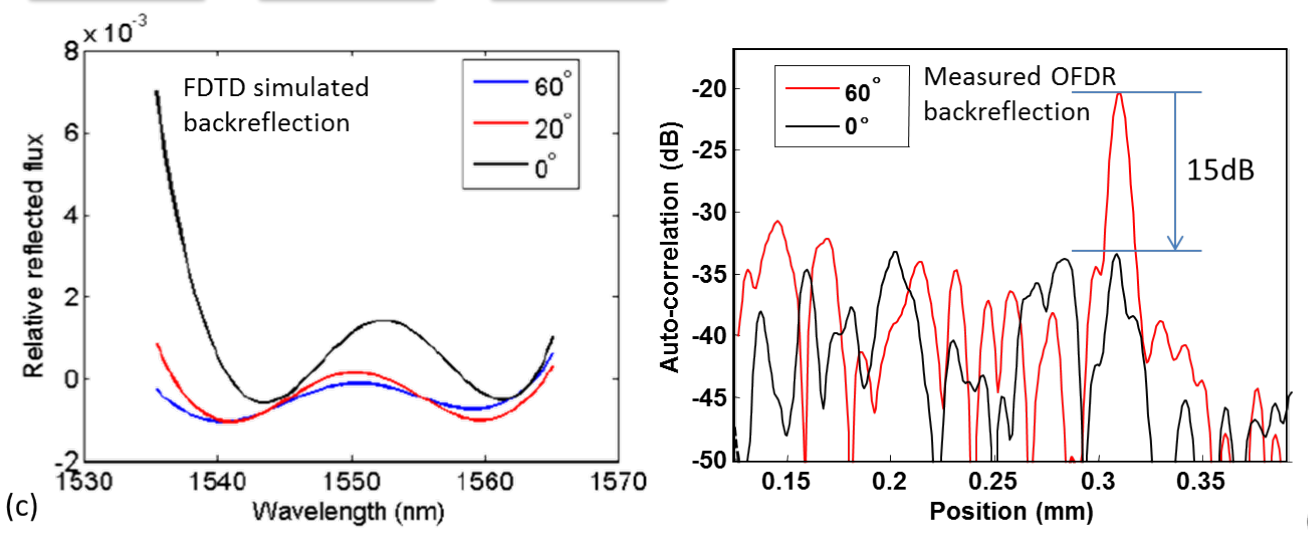

Figure 4. Optimizing a MMI-based 3dB combiner.

To design an MMI with lower backreflections, we could start from the existing component in the IPKISS library. Using standard Python syntax, it is easy to derive a new component which automatically inherits all the properties of the original MMI (subclassing). Then, we added additional parameters to the component which can be used to alter the layout, especially the position of the corner which causes the backreflection. Fig. 4 illustrates the angle $\theta$ which we included, amongst others, as a new parameter. With the new parameters, we then need to define a new layout. This takes a bit more work, as we have to provide the coordinates of the new shapes in the layout. For this, we add one function to our component description which overrides the layout definition in the original MMI component. Now we have a parameterized component, but we do not know what values will result in the lowest backreflection. Running a series of FDTD simulations is just a straightforward as the first simulation, but as we work in a scripting environment, we can just as well write a short for loop to perform a sweep over the relevant parameters. Alternatively, we can use any optimization tool that is available in Python, such as the python.optimize package. The results of this exercise are shown in Fig. 4: the simulation shown much less scattering at the corner, and the reflection is much lower with a tilted exit facet at $60^{\circ}{ }^{31}$ This is also experimentally confirmed. The optimized device was fabricated and characterized using OFDR measurement: backreflection was reduced with $15 \mathrm{~dB}$.

\subsection{An AWG with flat-band response}

A second example consists of an arrayed waveguide grating (AWG). This is a much larger component than the MMI, and it actually consists of an internal subcircuit. This is illustrated in Fig. 5. An input star coupler distributes the light over a set of waveguide apertures. These coupler into a bundle of delay lines, converging on a second star coupler. There, the light from the delay lines interferes to form an image that is captured by the output waveguides. The position and shape of the image is determined by the relative phases induced by the delay lines, and with the correct parameters, an AWG can serve as a wavelength demultiplexer, by coupling different wavelength channels to different output waveguides. ${ }^{25,32}$ In that function, it is often desirable that 


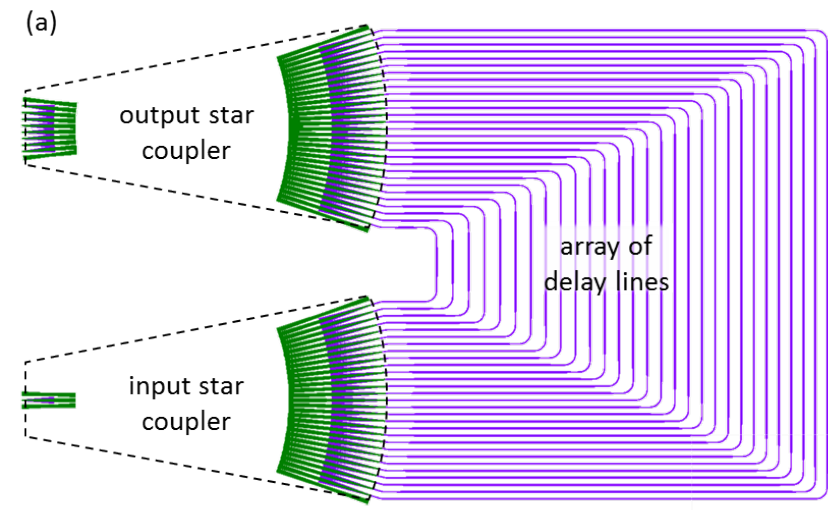

(b)
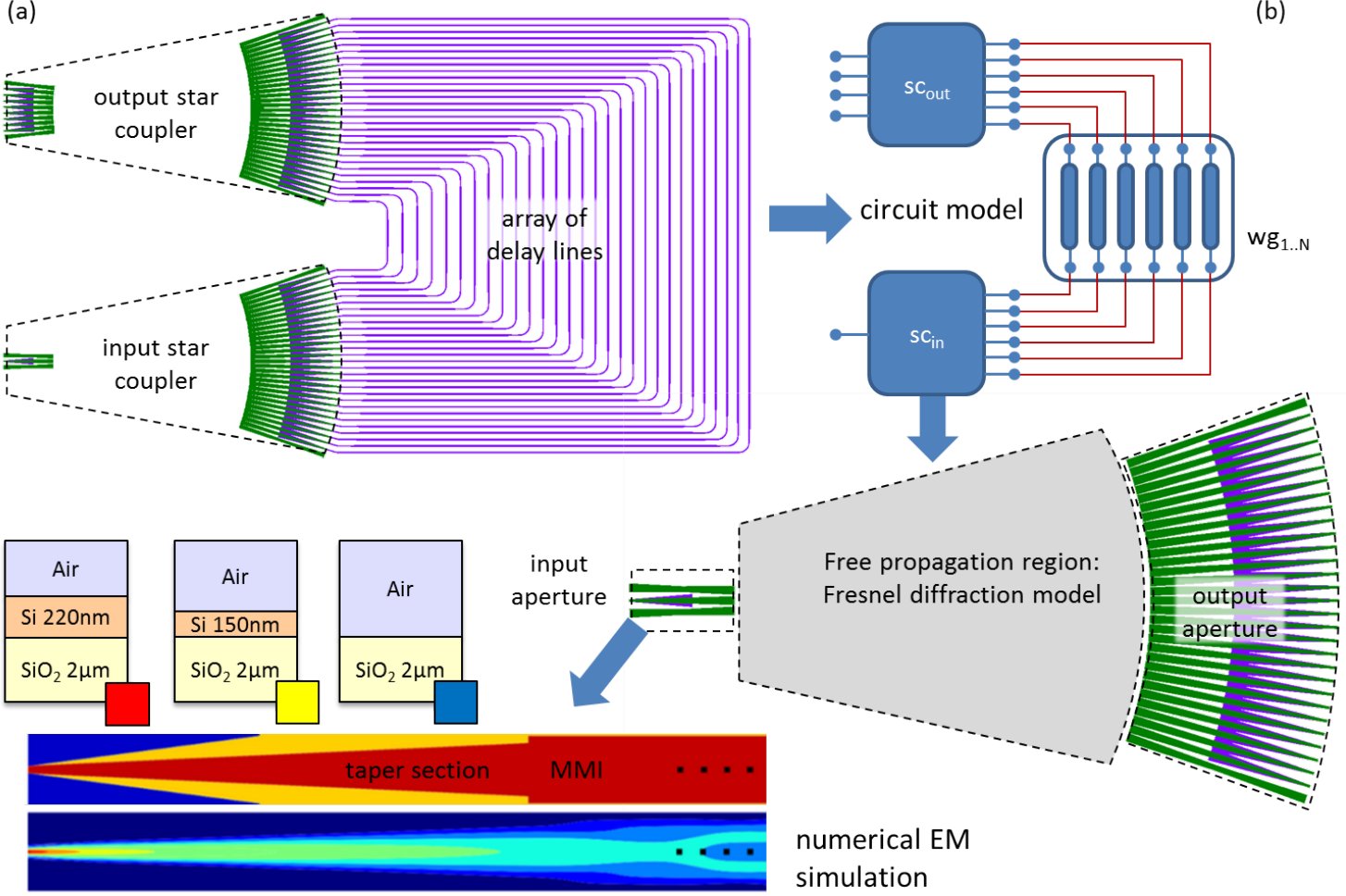

numerical EM

(d)

simulation

(c)

Figure 5. Breaking down an AWG (a) into subelements, each simulated with its own strategy. (b) The AWG can be respresented by circuit. The S-matrix of the delay lines is calculated analytically, while the star coupler (c) is broken down into separate electromagnetic simulations: A Fresnel diffraction model for the Free propagation region, and (d) an Eigenmode expansion or FDTD for the apertures.

the transmission spectrum at each output has a flat passband with a steep roll-off. This can be obtained by engineering the entrance and exit apertures in the star couplers. ${ }^{33,34}$

Simulating the response of an AWG is not straightforward: the device is too large to apply a direct FDTD simulation, and it also consists of very different parts. The hierarchy in IPKISS allows us to simulate each part with its own strategy, and link the total transmission together in a small circuit simulation. As an AWG is a passive linear component, we can perform the circuit simulation in the frequency domain, so it suffices to calculate wavelength-dependent scatter matrices (S-matrix) or transmission matrices (T-matrix) to model the entire circuit. The S-matrix for the individual delay is rather trivial: this is given by the length and the effective index (wavelength dependent). However, we include the possibility to incorporate phase errors, induced by sidewall roughness, which scales with the square root of the length of the waveguide. ${ }^{35}$ The star coupler itself consists of two types of structures. Tapered waveguides which end in an aperture, and an unpatterned free-propagation region (FPR). The latter is modeled as a homogeneous 2-D medium with an effective index using the Fresnel formalism, ${ }^{36}$ to calculate the field profile at the output apertures based on the field profile launched from the input aperture. More sophisticated modes can take into account the excitation of multiple modes in the unpatterned slab waveguides. ${ }^{37,38}$ The propagation in the apertures themselves is handled with an electromagnetic solver, and here again we can use different techniques. We could use 3-D FDTD as in the previous example, but in this case we found 2-D eigenmode expansion to be sufficient. By launching the ground mode of the waveguide toward the FPR, we obtain the field profile at the interface with the FPR, which we can use launch in the 2-D slab calculations, and which can (at the other side) be used to perform the overlap calculations to obtain the transmission at the outputs. The mix of these different techniques is managed by IPKISS. When we request a circuit model for the entire AWG, the respective simulations are triggered in the 

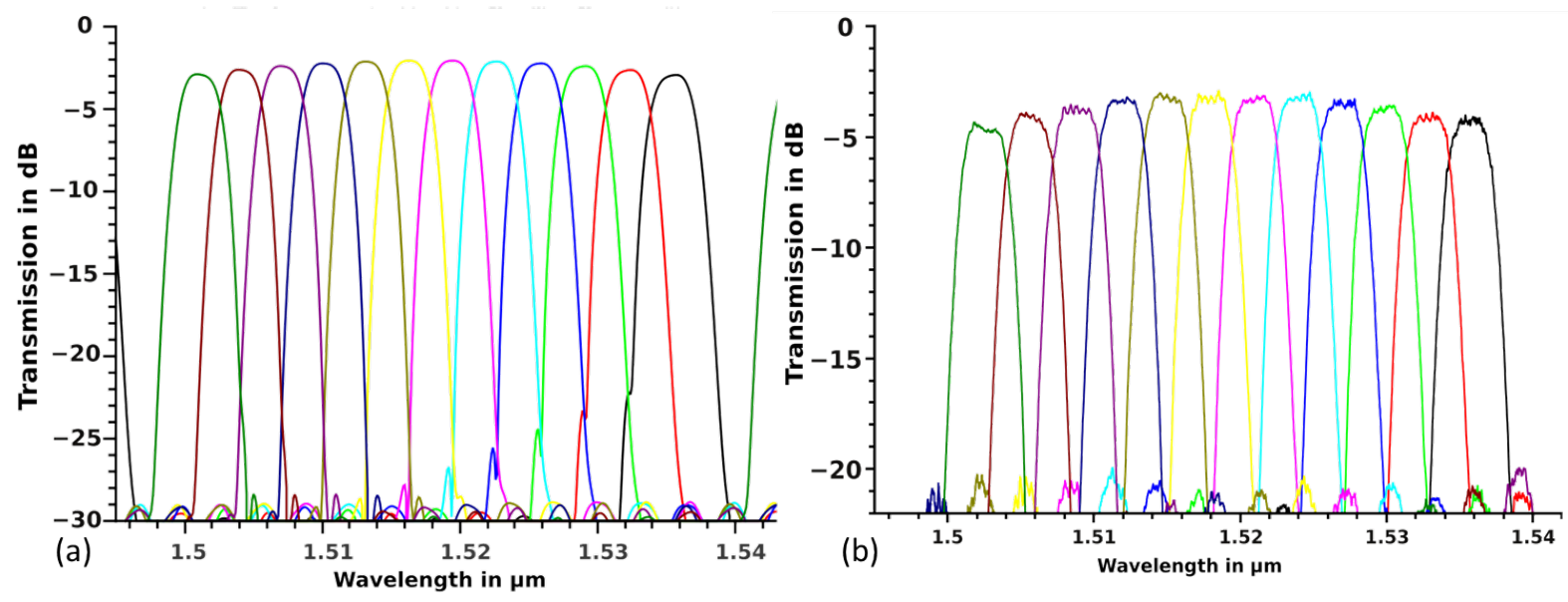

Figure 6. Transmission of AWG output channels for a device optimized for flat-top clannel response using an MMI entrance aperture. (a) simulation using the IPKISS design framework; (b) measured transmission of the fabricated device. The device is discussed in detail in. ${ }^{34}$

subcomponents. In principle, the parent component does not need to know what type of simulation will be triggered by its children. It only knows that it will get an S-matrix or T-matrix in return. This way, components can be replaced by better versions, or individual simulation strategies can be adapted, without impact on the simulation flow.

We optimized an AWG to obtain a flat-top spectral response by adding an MMI in the entrance aperture to tailor the field profile launched into the free propagation region. ${ }^{34}$ Because the AWG is hierarchical, we can replace the entrance waveguide aperture with a customized version. In our case, we did this again through subclassing, but the IPKISS framework provides several entry points to modify such hierarchical building blocks. the result is shown in Fig. 5. The pass-bands have a flatter top, compared to the regular parabolic profile. This design was also experimentally verified: the resulting transmission is also plotted. Details of this device are published in. ${ }^{34}$

\subsection{Synthesizing an MZI lattice filter from circuit to layout}

In this third example we construct a higher-order spectral filter based on Mach-Zehnder interferometers (MZI). ${ }^{39,40}$ Photonics lattice filters can be constructed using general purpose filter design algorithms. As MZIs do not contain any feedback, we can use finite-impulse response (FIR) filter synthesis. Based on the functional parameters (pass-band, free spectral range, filter order) and the windowing function (e.g. Chebychev). From this, we can calculate the optical delay lengths in the MZI stages, as well as the coupling coefficients. This can be fed into the Caphe circuit simulator, which can take into account the actual imperfections (e.g. higher-order dispersion) or stochastic variations (e.g. phase errors in the delay lines) to calculate the filter response. This is plotted in Fig. 7 .

The functional parameters such as coupling coefficients need to be translated into physical parameters for the design. Again, inheritance in IPKISS can be used to convert functional parameters to physical parameters. For instance, a directional coupler component could be described on a physical level, with parameters such as waveguide length, spacing, and bend radius. But it could be extended (through subclassing) with functional parameters (such as cross-coupling coefficient). A model can then be added to the component that converts these functional parameters to the physical ones. While such a model could invoke actual physical simulations (as shown in previous examples), for many components a reduced analytical expression, a fit or a lookup-table is used, where the parameters have been established through simulation or experimentally in advance. Through this mechanism, the filter layout is generated from the functional circuit description and exported to GDSII for fabrications. The measured results are also plotted in Fig. 7, and show a significant discrepancy with the design. This can be attributed to errors in the coupling sections. This illustrates that the design flow is only as good 


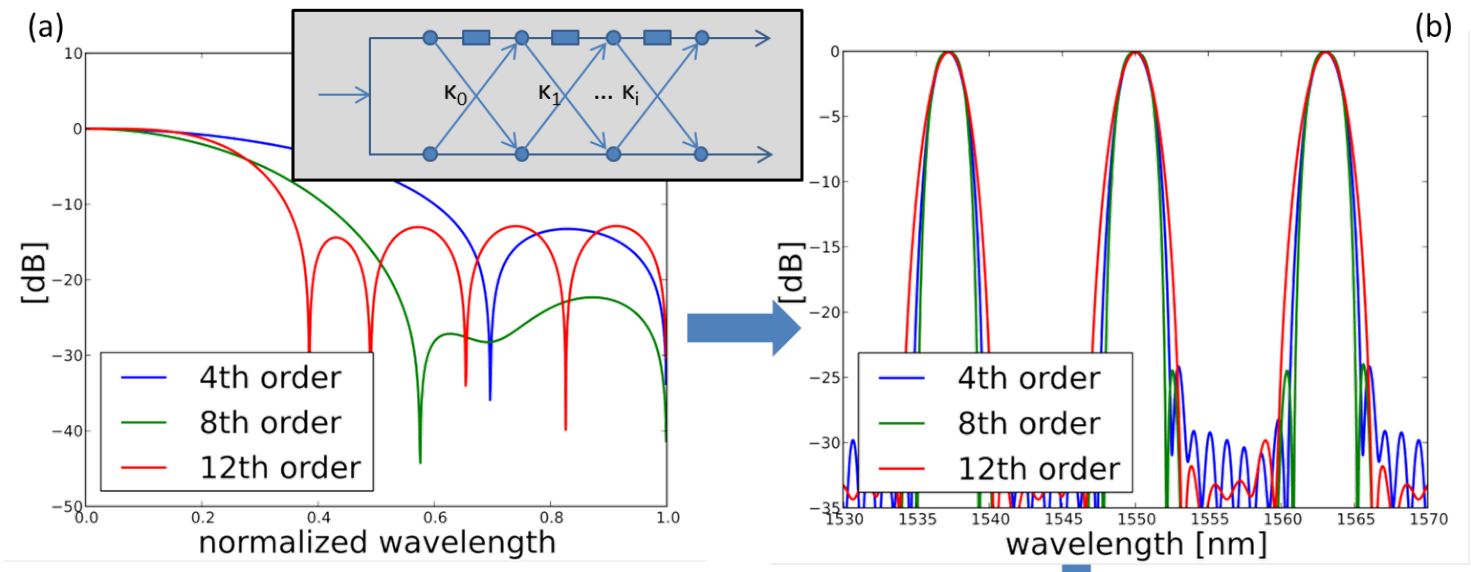

(b)

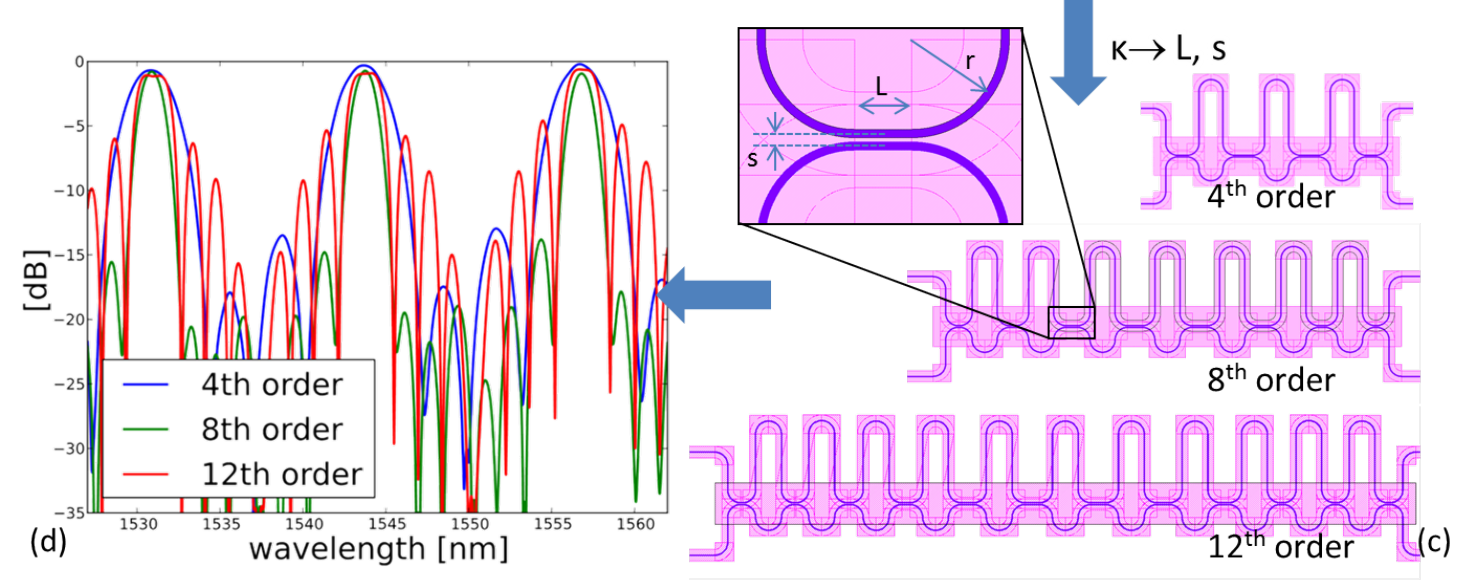

Figure 7. Designing lattice filter of different orders $(4,8,12)$. (a) Designing the filter response using standard filter synthesis techniques; (b) converting this to a wavelength response and simulating this as a circuit in Caphe, including waveguide nonidealities; (c) generating the physical layouts and (d) the final optical measurement result. The measurements show nonidealities because the coupler model is not sufficiently accurate.

as the models (either derived from physical simulations or supplied by the fab) that are being used. A likely cause is a mismatch between the manufactured line widths (due to a fabrication bias) and the values used in the design process.

\section{CONCLUSION}

We discussed the need for a photonic design flow which can provide access to the physical and the circuit level at the same time. Several tools exist in the market which provide a part of such a design flow. We presented our own design framework IPKISS, which is based on a somewhat different philosophy than most tools, in the sense that it is entirely based on user-side scripting in the industry-standard Python language. This makes the framework very flexible, which enables us to integrate a variety of simulation techniques within the same workflow: FDTD, eigenmode expansion on the physical level and optical circuit simulations through the interface with Caphe. ${ }^{22}$ IPKISS also makes it possible to generate complex, hierarchical mask layouts. We illustrated the functionality of IPKISS with a number of examples of design, physical and circuit modeling.

\section{REFERENCES}

[1] Oskooi, A. F., Roundy, D., Ibanescu, M., Bermel, P., Joannopoulos, J. D., and Johnson, S. G., "MEEP: A flexible free-software package for electromagnetic simulations by the FDTD method," Comp. Phys. Comm. 181, 687-702 (January 2010). 
[2] Lumerical Solutions, Inc., "Fdtd solutions." http://www.lumerical.com/tcad-products/fdtd/.

[3] Phoenix BV, "Photonic design automation." http://www.phoenixbv.com/.

[4] RSoft, "Photonics and network design software." http://www.rsoftdesign.com/.

[5] Optiwave, "Design software for photonics." http://www.optiwave.com.

[6] Photon Design, "FIMMProp-3D, a bidirectional optical propagation tool." http://www.photond.com/products/fimmprop.htm.

[7] Bienstman, P. and Baets, R., "Optical modelling of photonic crystals and vcsels using eigenmode expansion and perfectly matched layers," Opt. Quantum Electron. 33(4/5), 327 (2001).

[8] Scarmozzino, R., Gopinath, A., Pregla, R., and Helfert, S., "Numerical techniques for modeling guided-wave photonic devices," J. Sel. Top. Quantum Electron. 6(1), 150-162 (2000).

[9] Fiers, M., Van Vaerenbergh, T., Caluwaerts, K., Vande Ginste, D., Schrauwen, B., Dambre, J., and Bienstman, P., "Time-domain and frequency-domain modeling of nonlinear optical components at the circuit-level using a node-based approach," JOSA B 29(5), 896-900 (2012).

[10] Arellano, C., Mingaleev, S., Sokolov, E., Koltchanov, I., and Richter, A., "Time-and-frequency-domain modeling (tfdm) of hybrid photonic integrated circuits," in [SPIE OPTO], 82650K-82650K, International Society for Optics and Photonics (2012).

[11] Lumerical Solutions, Inc., "Interconnect." http://www.lumerical.com/tcad-products/interconnect/.

[12] Melati, D., Morichetti, F., Soares, F. M., and Melloni, A., "Building block based design of photonic integrated circuits for generic photonic foundries," in [Transparent Optical Networks (ICTON) 2012], 1-4, IEEE (2012).

[13] Bogaerts, W., Dumon, P., Fiers, M., Ribeiro, A., and Vanslembrouck, M., "Silicon photonics integrated design," in [International Conference on Fibre Optics and Photonics], Optical Society of America (2012).

[14] Arellano, C., Mingaleev, S., Koltchanov, I., Richter, A., Pomplun, J., Burger, S., and Schmidt, F., "Efficient design of photonic integrated circuits (pics) by combining device-and circuit-level simulation tools," in [SPIE OPTO], 862711-862711, International Society for Optics and Photonics (2013).

[15] Melati, D., Morichetti, F., Canciamilla, A., Roncelli, D., Soares, F., Bakker, A., and Melloni, A., "Validation of the building-block-based approach for the design of photonic integrated circuits," J. Lightwave Technol. 30(23), 3610-3616 (2012).

[16] Ghent University - imec, "IPKISS parametric design framework." http://www.ipkiss.org.

[17] Fiers, M., Lambert, E., Pathak, S., Dumon, P., Maes, B., Bienstman, P., and Bogaerts, W., "Improving the design cycle for nanophotonic components," J. Comp. Sci. to be published (2013).

[18] Mentor Graphics, "Calibre - IC verification." http://www.mentor.com.

[19] Design Workshop, "Dw2000 - photonic physical layout and verification software." http://www.designw.com/PDA-Overview.php.

[20] Filarete, "ASPIC photonic circuit simulator." http://www.aspicdesign.com/.

[21] Bienstman, P., Vanholme, L., Bogaerts, W., Dumon, P., and Vandersteegen, P., "Python in nanophotonics research," Comp. Sci. Eng. 9(3), 2801-2803 (2007).

[22] Ghent University - imec, "Caphe circuit simulator." http://www.caphesim.eu.

[23] Bogaerts, W., Bradt, P., Vanholme, L., Bienstman, P., and Baets, R., "Closed-loop modeling of silicon nanophotonics from design to fabrication and back again," Opt. Quantum Electron. 40, 801-811 (Sep. 2008).

[24] Selvaraja, S., Jaenen, P., Bogaerts, W., Van Thourhout, D., Dumon, P., and Baets, R., "Fabrication of photonic wire and crystal circuits in silicon-on-insulator using 193nm optical lithography," J. Lightwave Technol. 27(18), 4076-4083 (2009).

[25] Bogaerts, W., Selvaraja, S., Dumon, P., Brouckaert, J., De Vos, K., Van Thourhout, D., and Baets, R., "Silicon-on-insulator spectral filters fabricated with cmos technology," J. Sel. Top. Quantum Electron. 16(1), $33-44(2010)$.

[26] Bogaerts, W., De Heyn, P., Van Vaerenbergh, T., De Vos, K., Kumar Selvaraja, S., Claes, T., Dumon, P., Bienstman, P., Van Thourhout, D., and Baets, R., "Silicon microring resonators," Laser Photon. Rev. 6(1), $47-73$ (2012). 
[27] Taillaert, D., Bienstman, P., and Baets, R., "Compact efficient broadband grating coupler for silicon-oninsulator waveguides," Opt. Lett. 29, 2749-2751 (December 2004).

[28] Hui, Y., Pantouvaki, M., Van Campenhout, J., Korn, D., Komorowska, K., Dumon, P., Li, Y., Verheyen, P., Absil, P., Alloatti, L., Hillerkuss, D., Leuthold, J., Baets, R., and Bogaerts, W., "Performance tradeoff between lateral and interdigitated doping patterns for high speed carrier-depletion based silicon modulators," Opt. Express 20, 12926-12938 (Jun 2012).

[29] Dumon, P., Bogaerts, W., Baets, R., Fedeli, J.-M., and Fulbert, L., "Towards foundry approach for silicon photonics: silicon photonics platform epixfab," Electron. Lett. 45(12), 581-582 (2009).

[30] Li, Y., Verstuyft, S., Yurtsever, G., Keyvaninia, S., Roelkens, G., Van Thourhout, D., and Baets, R., "Heterodyne laser doppler vibrometers integrated on silicon-on-insulator based on serrodyne thermo-optic frequency shifters," Appl. Opt. 52(10), 2145-2152 (2013).

[31] Li, Y. and Baets, R., "Improved multi-mode interferometers (mmis) on silicon-on-insulator with the optimized return loss and isolation," in [16th Annual Symposium of the IEEE Photononics Benelux Chapter, Belgium], (2011).

[32] Dragone, C., "An NxN optical multiplexer using a planar arrangement of two star couplers," IEEE Photon. Technol. Lett. 3, 812-814 (Sept. 1991).

[33] Soole, J. B. D., Amersfoort, M., Leblanc, H., Andreadakis, N. C., Rajhel, A., Caneau, C., Bhat, R., Koza, M., Youtsey, C., and Adesida, I., "Use of multimode interference couplers to broaden the passband of wavelength-dispersive integrated wdm filters," Photon. Technol. Lett., 8(10), 1340-1342 (1996).

[34] Pathak, S., Vanslembrouck, M., Dumon, P., Van Thourhout, D., and Bogaerts, W., "Optimized silicon awg with flattened spectral response using an mmi aperture," J. Lightwave Technol. 31(1), 87-93 (2013).

[35] Goh, T., Suzuki, S., and Sugita, A., "Estimation of waveguide phase error in silica-based waveguides," $J$. Lightwave Technol. 15(11), 2107-2113 (1997).

[36] Doerr, C. R., "Planar lightwave devices for wdm," in [Optical Fiber Telecommunications], Kaminow, I. P. and Li, T., eds., IV A, ch. 9, 405 - 476, Academic Press, ISBN 0-12-395172-0 (2002).

[37] Lycett, R. J., Gallagher, D., and Brulis, V., "Perfect chirped echelle grating wavelength multiplexor: Design and optimization," IEEE Photon. J. 5(2), 2400123-2400123 (2013).

[38] Photon Design, "Epipprop, an echelle grating model." http://www.photond.com/products/epipprop.htm.

[39] Bogaerts, W., Dumon, P., Van Thourhout, D., Taillaert, D., Jaenen, P., Wouters, J., Beckx, S., Wiaux, V., and Baets, R., "Compact wavelength-selective functions in silicon-on-insulator photonic wires," J. Sel. Top. Quantum Electron. 12, 1394-1401 (December 2006).

[40] Yamada, K., Shoji, T., Tsuchizawa, T., Watanabe, T., Takahashi, J., and Itabashi, S., "Silicon-wire-based ultrasmall lattice filters with wide free spectral ranges," J. Sel. Topics Quantum Electron 11, 232-240 (2005). 